\title{
El impacto del Brexit en la economía española*
}

\section{The impact of Brexit on the Spanish economy}

\author{
María C. Latorre \\ Universidad Complutense de Madrid \\ Hidemichi Yonezawa \\ Statistics Norway
}

\begin{abstract}
Resumen
Estimamos los efectos micro y macroeconómicos del Brexit utilizando un modelo de equilibrio general aplicado. Simulamos varios escenarios: salida sin acuerdo, permanencia de Reino Unido en la unión aduanera europea, propuesta de Brexit de Boris Johnson, Brexit blando y combinación del Brexit de Boris Johnson con un posible tratado con EEUU. El modelo incluye heterogeneidad empresarial à la Melitz (2003) y multinacionales en servicios. El impacto para España es limitado, aunque conforme más lejana sea la relación futura (y, en consecuencia, mayores barreras al comercio y a la inversión extranjera directa surjan) el Brexit resulta más dañino.

Palabras clave: estimaciones empíricas, comercio internacional, multinacionales, inversión extranjera directa, modelo de equilibrio general computable, integración económica.
\end{abstract}

Clasificación JEL: C68, F14, F15, F17, F23.

\begin{abstract}
We estimate the micro and macroeconomic effects of Brexit using a computable general equilibrium (CGE) model. We simulate several scenarios: exit without agreement, UK stay in the European customs union, Boris Johnson's Brexit proposal, soft Brexit and a combination of Boris Johnson's Brexit with a possible treaty with the US. The model includes business heterogeneity à la Melitz (2003) and multinationals in services. The impact for Spain is limited, although the more distant the future relationship is (i.e., the higher the barriers to trade and foreign direct investment are) the more damaging Brexit becomes.
\end{abstract}

Keywords: empirical estimates, international trade, multinational companies, foreign direct investment, computable general equilibrium model, economic integration.

JEL Classification: C68, F14, F15, F17, F23.

\section{Introducción}

Desde el 1 de enero de 2021 la relación de la UE con Reino Unido será necesariamente diferente a la que desde hace décadas ambas partes han ido entretejiendo. A pesar de que ya desde enero de 2020 se ha iniciado un periodo transitorio para

* Este trabajo está basado en un estudio realizado para el Ministerio de Industria, Comercio y Turismo. 
negociar la forma final que esa separación tendrá, las consecuencias económicas de la misma comenzarán en 2021. Ni siquiera la crisis generada por la COVID-19 ha animado al Gobierno británico a pedir una extensión para contar con más tiempo para negociar la futura relación. Hasta dos años adicionales podían haber solicitado para el periodo transitorio, un tiempo que sigue siendo muy limitado considerando lo que suelen necesitar los tratados comerciales entre países. Recordemos que las negociaciones de la UE con Canadá para un tratado como el CETA llevaron seis años, y es algo mucho más sencillo que el entramado de relaciones existentes en la UE.

El Gobierno británico que surgió con Boris Johnson como primer ministro en diciembre de 2019, cuenta con una enorme mayoría en el Parlamento y tiene prisa por implementar la ruptura con la UE. Están convencidos de los beneficios económicos de esa ruptura, algo que la mayoría de los economistas ponen en entredicho (Bisciari, 2019; Latorre et al., 2020a; Latorre et al., 2019; Fernández-Pacheco et al., 2018, Busch y Matthes, 2016). Además, a tres meses de la conclusión del periodo transitorio, el proceso de negociación del Brexit ha pasado por serias dificultades. Mediante el proyecto de la Ley del Mercado Interior del Reino Unido el Gobierno británico trata de arrogarse dos decisiones que violan el protocolo acordado para Irlanda del Norte en el Acuerdo de Retirada firmado por el propio Boris Johnson. El Gobierno británico decidiría: 1) si comunicar o no a Bruselas subvenciones públicas a empresas radicadas en Irlanda del Norte; y 2) qué mercancías deberían someterse a declaración de aduanas en los intercambios entre Irlanda del Norte y el resto del Reino Unido. Nótese que el protocolo de Irlanda del Norte debe respetarse independientemente de que la UE y Reino Unido alcancen o no un acuerdo que guíe sus relaciones en la era post-Brexit. Aunque la UE ha emprendido acciones judiciales y la reputación internacional de Reino Unido debería quedar dañada por este episodio, las negociaciones se han retomado.

El Brexit tendrá consecuencias para el comercio y la inversión extranjera directa (IED) que la economía española emite y recibe. Supone poner trabas a estos flujos, lo que influirá, a su vez, en numerosas variables macro y microeconómicas en España.

España destina aproximadamente el $8 \%$ de sus exportaciones agregadas a Reino Unido y realiza aproximadamente un $5 \%$ del total de sus importaciones desde este país (cálculos para el total de bienes y servicios). Por su parte, las multinacionales españolas operando en sectores de servicios ${ }^{1}$ en Reino Unido explican, aproximadamente, el $2 \%$ de las ventas totales en los sectores de servicios de ese país, mientras que las multinacionales británicas explican aproximadamente el 5,14 \% de las ventas en España de los sectores de servicios. Cifras que esconden una considerable variación en los distintos sectores de la economía española y requieren de otros matices importantes.

${ }^{1}$ Estos cálculos se basan en el peso de las multinacionales en lo que en la literatura se suele denominar «servicios avanzados», que son en los que incluimos multinacionales en nuestro modelo. Los porcentajes concretos de pesos de multinacionales que citamos aquí, por distintas razones, no incluyen los servicios públicos, ni varios servicios de red como la provisión de energía, ni el comercio al por mayor y por menor, ni los servicios financieros. 
Nuestros exportadores tendrán mayor dificultad para acceder al mercado británico y, según nuestras estimaciones, el volumen agregado de las exportaciones se contraería. A nivel sectorial las reducciones en las exportaciones serían muy heterogéneas dependiendo fundamentalmente de: 1) el tamaño de barreras que surjan con el Brexit, que son distintas por sectores; 2) el clima de competencia en el sector; 3) la importancia de Reino Unido en el total de destinos de sus exportaciones; y 4) el peso de las exportaciones en el total de los posibles usos de la producción sectorial.

Así mismo, en las importaciones españolas predominan las de bienes y servicios (inputs) intermedios. Esto implica que se trata más bien de importaciones necesarias para la producción de nuestra economía y no tanto competencia para las empresas españolas. Con el Brexit experimentarán más trabas que antaño, lo cual las encarecería, generando un aumento de costes para las empresas que dependen de ellas, si no son fácilmente sustituibles por productos domésticos o por importaciones procedentes de otros países.

Nuestras estimaciones y la de la mayoría de los trabajos sugieren que el Brexit reducirá los flujos de exportaciones españolas hacia Reino Unido y las importaciones provenientes de este país, así como las operaciones de las multinacionales españolas en Reino Unido y de las británicas en España. Esta reducción de la actividad económica causaría una contracción de la producción, los salarios y el empleo en España.

Aunque el daño global para la economía española parece limitado, las estimaciones varían en función del acuerdo final respecto a la relación futura tras el Brexit. Conforme más lejana sea la relación futura (y, en consecuencia, surjan mayores barreras al comercio y a la inversión extranjera directa) el Brexit resultaría más dañino.

En este trabajo ofrecemos las estimaciones del impacto micro y macroeconómico del Brexit en la economía española, utilizando un modelo de equilibrio general aplicado. Desplegamos resultados para los que, hasta donde podemos saber tras haber revisado la literatura sobre el Brexit (Latorre et al., 2020a) no existe evidencia previa, tales como la evolución de la producción, el empleo y los salarios a nivel sectorial, así como un amplio elenco de estimaciones macroeconómicas. Consideramos los siguientes escenarios: salida sin acuerdo, permanencia de Reino Unido en la unión aduanera europea, propuesta de Brexit de Boris Johnson, Brexit blando y combinación del Brexit de Boris Johnson con un posible tratado con EEUU. El modelo incluye heterogeneidad empresarial à la Melitz (2003) y multinacionales en los sectores de servicios.

Son pocos los trabajos que han analizado el impacto del Brexit en la economía española (Dhingra et al., 2017; Felbermayr et al., 2018; Vega, 2019; Hurtado, 2019). El consumo privado es una variable común en todo ellos, por lo que permite comparar los resultados. Dhingra et al. (2017) analizan los cambios en el bienestar para dos escenarios de Brexit (blando y duro) muy similares a los de este trabajo. Miden el bienestar con la variación en el consumo real per cápita que, dado el lento crecimiento poblacional en Reino Unido y la UE, supone una tasa de variación muy cercana 
a la del propio consumo real de este trabajo. Utilizan un New Quantitative Trade Model (NQTM), en el que la producción tiene lugar en un clima de competencia perfecta y que no incluye efectos de la IED.

Felbermayr et al. (2018) también analizan el impacto en el consumo real de un Brexit blando y duro para España muy similar al de este trabajo. Su definición de Brexit blando difiere de la que modelizamos aquí porque se trataría de una relación entre el Reino Unido y el resto de la UE como la del Acuerdo de libre comercio de 2011 entre la UE y la República de Corea. Su Brexit duro es como el que modelizamos en este estudio, con la salvedad de que Reino Unido dejaría de tener activos todos los acuerdos comerciales con terceros países que ahora tiene como miembro de la UE, y desaparecerían sus contribuciones al presupuesto europeo. La metodología también es un NQTM en el que la producción tiene lugar en un marco de competencia perfecta, sin considerar el impacto de la IED.

Hurtado (2019) actualiza alguna de las últimas estimaciones de los efectos que Vega (2019) realiza para el Banco de España a través de su Modelo Trimestral del Banco de España. Al comentar que la producción se realiza con una función de producción Cobb-Douglas, se puede inferir que estamos en un contexto de rendimientos constantes a escala, que difiere de la competencia imperfecta con rendimientos crecientes para la mayoría de los sectores presentes en este trabajo. Si bien, el modelo del Banco de España enriquece su función de producción no sólo tomando en cuenta los salarios y el stock de capital, sino incluyendo también la población en edad de trabajar y el crédito en sus análisis, aunque no parece considerar los bienes intermedios. Tanto en Hurtado (2019) como en Vega (2019) se recogen tres escenarios: un Brexit blando y dos tipos de Brexit duro. El Brexit blando modeliza una transición ordenada con un periodo transitorio relativamente largo y un acuerdo como el CETA con Canadá de 2016, por lo que difiere del de los trabajos previos y del de este trabajo. Por otra parte, el primer Brexit duro representa también una salida ordenada, pero las reglas de intercambio serían las que existen entre socios de la OMC, por lo que sería el más comparable con el de este trabajo y el de Dhingra et al. (2017) y el de Felbermayr et al. (2018). El segundo Brexit duro es igual al anterior, excepto en que la salida no es ordenada y se producen disrupciones en las cadenas productivas y hay inestabilidad financiera. Hurtado (2019) y Vega (2019) utilizan un modelo macroeconométrico con mecanismo de corrección del error para previsiones a medio plazo de la economía española (estimaciones a 5 años), con representación de los canales de demanda.

Resumimos todos los resultados en el Cuadro 1, el cual refleja que al eliminar las estructuras de Melitz y Krugman de nuestro modelo, los resultados se acercarían a los de los del resto de trabajos. Cuando damos un paso más y eliminamos también la IED, los modelos son cada vez más similares a nivel macroeconómico. Con todo, conviene no olvidar que no podemos comparar los resultados sectoriales porque el resto de metodologías consideradas en el el cuadro no los deriva. 


\section{CUADRO 1}

\section{RESUMEN DE LOS EFECTOS DEL BREXIT SOBRE EL CONSUMO EN ESPAÑA} (En \%)

\begin{tabular}{|l|c|c|}
\hline & Brexit blando & Brexit duro \\
\hline Dhingra et al. (2017) & $-0,07$ & $-0,20$ \\
\hline Felbermayr et al. (2018) & $-0,13$ & $-0,39 /-0,42$ \\
\hline Vega (2019) y Hurtado (2019) & $-0,01$ & $-0,24 /-0,35$ \\
\hline $\begin{array}{l}\text { Latorre y Yonezawa (presente estudio con competencia } \\
\text { monopolística à la Melitz e IED) }\end{array}$ & $-0,54$ & $-1,08$ \\
\hline $\begin{array}{l}\text { Latorre y Yonezawa (modelo de este estudio operando en } \\
\text { competencia perfecta con IED) }\end{array}$ & $-0,30$ & $-0,62$ \\
\hline $\begin{array}{l}\text { Latorre y Yonezawa (modelo de este estudio operando en } \\
\text { competencia perfecta y sin IED) }\end{array}$ & $-0,17$ & $-0,41$ \\
\hline
\end{tabular}

FUENTE: Dhingra et al. (2017), Felbermayr et al. (2018), Vega (2019), Hurtado (2019) y resultados de este trabajo.

\section{Modelo, datos y simulaciones}

Como acabamos de explicar, para estimar el impacto del Brexit utilizamos un modelo de equilibrio general computable. También llamado Modelo de Equilibrio General Aplicado (MEGA) o Computable General Equilibrium (CGE) Model. Este modelo cuenta con el respaldo de varias publicaciones científicas (Latorre et al., 2020a; 2020b) y podríamos decir que se encuentra en la frontera de la ciencia por su combinación única de dos características avanzadas. Estas dos características son: 1) la modelización de un clima de competencia à la Melitz (2003) en varios sectores manufactureros; y 2) la presencia de multinacionales extranjeras en los sectores de servicios avanzados, operando en un clima de competencia imperfecta à la Krugman (1980). Esta modelización supone un importante paso al frente no sólo respecto a otras metodologías como acabamos de comentar en la Introducción, sino también respecto a otros MEGA que utilizan un clima de competencia perfecta, sin considerar la presencia de la IED (Shoven y Whalley, 1992; Burfisher, 2016), como señalan Dixon y Jorgenson (2013).

Al incluir un clima de competencia monopolística à la Melitz (2003) el modelo permite captar dos efectos del mundo real: 1) la existencia de empresas con tamaños, costes, productividades y precios distintos dentro de cada sector, lo que genera variaciones endógenas en la productividad cuando surgen barreras al comercio; y 2) la capacidad de captar que las empresas abran o cierren, o que dejen de servir un mercado extranjero para pasar a servir en otro. Es decir, Melitz captura variaciones no sólo en el margen intensivo del comercio internacional (variaciones en su intensidad para un determinado origen o destino), sino también en el margen extensivo (o cambios en el número de países de destino u origen de los flujos comerciales). 
Adicionalmente, para la modelización de la economía española en el presente trabajo hemos introducido la presencia de desempleo (usando una wage curve siguiendo a Latorre et al., 2015), algo que no habíamos hecho en los trabajos de Latorre et al. (2020a; 2020b). Esta modelización recoge la relación de que, a mayor tasa de desempleo, menor debería ser el salario y viceversa. También hemos introducido fricciones en la movilidad de la mano de obra (la mitad de la mano de obra de cada sector es específica de ese sector y no se puede desplazar a otros sectores).

Es importante señalar que los resultados del MEGA aíslan y cuantifican el impacto del shock concreto que se analiza. En otras palabras, el modelo estima los efectos del Brexit, mientras que, en el mundo real, el Brexit interactuará con otras muchas fuerzas económicas que tendrán lugar a la vez (tales como el COVID, políticas fiscales, monetarias, comerciales...) que conllevan otros impactos simultáneos. Nuestros resultados se concentran en lo que cabe esperar del Brexit en sí mismo, independientemente de otras fuerzas que estén en marcha cuando este tenga lugar. No cabe interpretarlos como proyecciones.

Nuestro modelo divide la economía mundial en 22 sectores, 5 regiones (Reino Unido, España, el resto de la Unión Europea, los EEUU y el resto del mundo) y cuatro factores de producción (tierra, recursos naturales, capital, trabajo). Utiliza los datos de la última base de datos del GTAP 10 (Aguiar et al., 2019), aunque hemos realizado una proyección de los datos al año 2021 siguiendo el procedimiento descrito en Böhringer et al. (2009). GTAP es una de las escasas bases de datos disponibles para MEGA. En su última edición recoge la economía mundial para los años 2004, 2007, 2011 y 2014, dividida en 121 países y 20 regiones desagregados en 65 sectores que representan el $98 \%$ del PIB mundial, con información que cubre el marco input-output y un pormenorizado seguimiento de los flujos comerciales.

Las barreras al comercio que incorporamos en este trabajo siguen las del influyente análisis del Brexit de Dhingra et al. (2017), mientras que las barreras a las operaciones de las multinacionales se toman de Jafari y Tarr (2017). Para el tratado con EEUU las barreras siguen las del trabajo clásico de Francois et al. (2013). Además, los datos sobre la participación de las multinacionales y las empresas nacionales en las ventas de los sectores de servicios en cada una de las regiones del modelo proceden principalmente de Eurostat (2019). Véase Latorre et al. (2020b) para más detalles.

Estimamos el impacto de los siguientes escenarios:

- Brexit duro: salida de Reino Unido de la Unión Europea (UE) sin acuerdo, en la que los intercambios quedarían sujetos a las reglas de la Organización Mundial de Comercio. Supondría la adopción de aranceles de nación más favorecida (NMF) entre la UE y Reino Unido, y las mayores barreras no arancelarias al comercio y a la IED que pueden darse en la futura relación entre Reino Unido y España, por una parte, y entre Reino Unido y el resto de la UE (UE28, excluyendo España y Reino Unido), por otra. Si durante el periodo de transición, que durará hasta el 31 de diciembre de 2020, no se alcanzara un acuerdo, existiría 
la posibilidad de caer en este escenario de no deal, máxime con las recientes tensiones generadas por el órdago de la Ley del Mercado Interior del Reino Unido. Adicionalmente, este escenario es una referencia importante para considerar las mayores pérdidas que cabría esperar del Brexit, según la mayoría de estudios económicos.

- Unión aduanera: salida de Reino Unido del mercado único europeo, pero permaneciendo en la unión aduanera. Esto implicaría mantener los aranceles entre las dos partes a cero y aranceles exteriores comunes, pero con aumentos en barreras no arancelarias elevadas al comercio y a la IED. Dado que la unión aduanera obligaría a Reino Unido a adoptar el arancel común que la UE dictara frente al exterior en los distintos sectores, parece poco probable que Reino Unido quiera ceder autonomía para establecer políticas comerciales frente a otros países. Esa capacidad de poner en marcha nuevas políticas comerciales, al margen de la UE, ha sido uno de los argumentos esgrimidos por los partidarios del Brexit. Nótese que cuando hablamos de aranceles, por ejemplo, en este contexto frente a terceros países, se sobreentiende que también tenemos en cuenta las cuotas, que están incluidas también en el cálculo de los aranceles de nuestro modelo.

- Brexit de Boris Johnson: relación basada en lo recogido en la «declaración política revisada» (Gov UK, 2019). Al tratarse a grandes rasgos de una propuesta de salida de Reino Unido de la unión aduanera ${ }^{2}$, para pasar a adoptar un tratado de libre comercio en el futuro con la UE, los aranceles serían nulos pero surgirán barreras no arancelarias entre Reino Unido y la UE. Se trata de una propuesta que implica mayores barreras que las que se deducen de la declaración política que había acordado la primera ministra, Theresa May.

- Brexit blando: En todos los escenarios previos los incrementos en barreras no arancelarias tanto para el comercio como para la IED serán elevados. Por este motivo, parece adecuado también ofrecer unas estimaciones de un acuerdo con una relación más cercana con la UE. La relación con las menores trabas posibles para terceros países es la del Espacio Económico Europeo, que habitualmente se ha modelizado siguiendo la situación de Noruega respecto a la UE. La relación futura definitiva entre Reino Unido y la UE está por definir y, aunque parece improbable que Reino Unido busque una relación muy cercana, debido a la obligación de aceptar inmigrantes, de aplicación de la regulación europea, sin poder votarla, y contribuciones al presupuesto que asume Noruega, este escenario es habitualmente un referente para el daño mínimo que el Brexit podría causar.

${ }^{2}$ La única excepción es el protocolo para Irlanda del Norte, que sería la única región británica que permanecería en la unión aduanera, durante el periodo transitorio hasta que se acordase una nueva relación ad hoc para esta región. La Ley del Mercado Interior del Reino Unido viola cláusulas propias de la unión aduanera europea. Irlanda del Norte equivale tan sólo el $2 \%$ del PIB de Reino Unido en 2017 y al 2,63 \% y $1,63 \%$ de las exportaciones e importaciones británicas, respectivamente, en 2018. 
- Brexit de Boris Johnson combinado con un tratado que reduzca las barreras al comercio y a la IED con EE UU: con dos variantes, entre las que se encuentran un tratado «profundo» (acuerdo ambicioso) y con un tratado con menores reducciones en las barreras (acuerdo modesto). Aunque hemos modelizado el impacto de que sea solo Reino Unido quien firme el acuerdo con EEUU, este es tan reducido para la economía española, que presentamos el impacto de un tratado con EEUU que lo firmaran de manera simultánea tanto Reino Unido como el resto de la UE (incluida España, por supuesto), con las mismas condiciones, ya sean modestas o ambiciosas.

\section{Resultados}

\subsection{Resultados macroeconómicos}

El Cuadro 2 recoge el impacto en las variables macroeconómicas de la economía española en sus columnas: PIB, consumo privado, salarios, empleo, exportaciones e importaciones. Se trata de un análisis muy detallado que pocos estudios sobre el Brexit ofrecen. En sus filas aparecen los cuatro bloques de escenarios del Brexit: duro, unión aduanera, el Brexit de Johnson y blando, junto con dos bloques finales que combinan el Brexit de Johnson con los efectos de un tratado, ya sea modesto o ambicioso con EE UU, de toda la UE de los 28 (incluyendo, por tanto, Reino Unido y España). Los resultados se expresan como porcentajes de variación respecto al nivel inicial de las variables (es decir, respecto a sus datos iniciales, o benchmark, a $1 \mathrm{de}$ enero de 2021).

Los escenarios y componentes del Cuadro 2 reflejan que en este trabajo nos centramos en dos efectos clave del Brexit: 1) las barreras que surgirán al comercio bilateral entre Reino Unido y España (y el resto de la UE). Estas barreras pueden ser barreras no arancelarias (BNA) o aranceles; 2) Las barreras a la actividad de las de filiales de multinacionales españolas (y del resto de la UE) que operan en Reino Unido y a la de las filiales de multinacionales de Reino Unido que están en España (y en el resto de la UE). Se trata también de BNA que ahora se aplican, sin embargo, a las operaciones de las multinacionales o a la IED. El lector interesado puede encontrar otros impactos más allá del comercio y la inversión extranjera directa en varias revisiones de la literatura (Bisciari, 2019; Latorre et al., 2019; Latorre et al., 2020a; Fernández-Pacheco et al., 2018; Busch y Matthes, 2016), que incluyen tanto MEGA aplicados al Brexit, como otras metodologías.

Así, para cada uno de estos escenarios se presenta de manera separada el impacto del surgimiento de barreras no arancelarias al comercio (fila de «BNA»), BNA a las operaciones de las multinacionales que, para diferenciarlas de las anteriores BNA al comercio, aparecen con la etiqueta de «IED» en los Cuadros y una fila que recoge el «Total» con el impacto de todas las barreras operando simultáneamente. En el caso del Brexit duro y de la unión aduanera se considera además el efecto de los 


\section{CUADRO 2}

IMPACTO MACROECONÓMICO DE LOS DISTINTOS ESCENARIOS

Y COMPONENTES DEL BREXIT Y DE UN ACUERDO DE LA UE Y REINO UNIDO CON EE UU TRAS EL BREXIT DE JOHNSON

(España, \% de variación respecto al nivel inicial)

\begin{tabular}{|c|c|c|c|c|c|c|}
\hline & PIB & $\begin{array}{c}\text { Consumo } \\
\text { privado }\end{array}$ & Salarios & Empleo & $\begin{array}{l}\text { Exporta- } \\
\text { ciones }\end{array}$ & $\begin{array}{c}\text { Importa- } \\
\text { ciones }\end{array}$ \\
\hline \multicolumn{7}{|c|}{ Brexit duro } \\
\hline Total & $-0,64$ & $-1,08$ & $-0,55$ & $-0,36$ & $-1,31$ & $-1,42$ \\
\hline Aranceles & $-0,11$ & $-0,19$ & $-0,17$ & $-0,08$ & $-0,52$ & $-0,54$ \\
\hline BNA & $-0,31$ & $-0,52$ & $-0,38$ & $-0,20$ & $-0,94$ & $-0,97$ \\
\hline IED & $-0,25$ & $-0,43$ & $-0,04$ & $-0,11$ & $-0,06$ & $-0,14$ \\
\hline \multicolumn{7}{|c|}{ Unión aduanera } \\
\hline Total & $-0,52$ & $-0,87$ & $-0,38$ & $-0,28$ & $-0,92$ & $-1,02$ \\
\hline Aranceles & $-0,02$ & $-0,03$ & $-0,04$ & $-0,01$ & $-0,12$ & $-0,12$ \\
\hline BNA & $-0,27$ & $-0,45$ & $-0,31$ & $-0,17$ & $-0,82$ & $-0,84$ \\
\hline IED & $-0,25$ & $-0,43$ & $-0,04$ & $-0,11$ & $-0,06$ & $-0,14$ \\
\hline \multicolumn{7}{|c|}{ Brexit de Johnson } \\
\hline Total & $-0,47$ & $-\mathbf{0 , 8 0}$ & $-0,33$ & $-0,26$ & $-\mathbf{0 , 8 3}$ & $-0,92$ \\
\hline BNA & $-0,25$ & $-0,42$ & $-0,30$ & $-0,16$ & $-0,77$ & $-0,80$ \\
\hline IED & $-0,23$ & $-0,39$ & $-0,04$ & $-0,10$ & $-0,05$ & $-0,13$ \\
\hline \multicolumn{7}{|c|}{ Brexit blando } \\
\hline Total & $-0,32$ & $-0,54$ & $-0,23$ & $-0,17$ & $-0,56$ & $-0,63$ \\
\hline BNA & $-0,17$ & $-0,28$ & $-0,20$ & $-0,11$ & $-0,53$ & $-0,55$ \\
\hline IED & $-0,16$ & $-0,26$ & $-0,03$ & $-0,06$ & $-0,03$ & $-0,08$ \\
\hline \multicolumn{7}{|c|}{ Brexit de Johnson + Tratado ambicioso de Reino Unido y la Unión Europea con EE UU } \\
\hline Total & $\mathbf{0 , 0 3}$ & $\mathbf{0 , 0 4}$ & $\mathbf{0 , 0 9}$ & $-0,04$ & $\mathbf{0 , 1 8}$ & $\mathbf{0 , 0 8}$ \\
\hline Aranceles & $-0,43$ & $-0,73$ & $-0,30$ & $-0,23$ & $-0,66$ & $-0,74$ \\
\hline BNA & $-0,10$ & $-0,17$ & 0,05 & $-0,10$ & $-0,17$ & $-0,22$ \\
\hline IED & $-0,40$ & $-0,67$ & $-0,32$ & $-0,24$ & $-0,74$ & $-0,90$ \\
\hline \multicolumn{7}{|c|}{ Brexit de Johnson + Tratado modesto de Reino Unido y la Unión Europea con EE UU } \\
\hline Total & $-0,27$ & $-0,46$ & $-0,18$ & $-0,17$ & $-0,37$ & $-0,45$ \\
\hline Aranceles & $-0,43$ & $-0,73$ & $-0,30$ & $-0,23$ & $-0,66$ & $-0,74$ \\
\hline BNA & $-0,35$ & $-0,59$ & $-0,20$ & $-0,20$ & $-0,60$ & $-0,68$ \\
\hline IED & $-0,44$ & $-0,74$ & $-0,33$ & $-0,25$ & $-0,79$ & $-0,91$ \\
\hline
\end{tabular}

FUENTE: Elaboración propia. 
aranceles, a los que se dedica otra fila. Recuérdese que en el Brexit duro, se trata del efecto conjunto de los aranceles de nación más favorecida para los distintos sectores de bienes. Los aranceles de la unión aduanera afectan únicamente a agricultura y alimentación y son los que España tiene en su unión aduanera con Turquía. También presentamos una fila que recoge el «Total» con el impacto de todas las barreras operando simultáneamente. El impacto total del Brexit duro y de la unión aduanera incorpora también los efectos de sus correspondientes aranceles.

El Brexit supone una fuerza contractiva de la actividad económica, que tenderá a reducir el crecimiento del PIB, del consumo privado, de los salarios, del empleo y del comercio internacional. Esto ocurre porque el comercio y las operaciones de las multinacionales caen, reduciendo las exportaciones y la producción, aunque, como se verá, un buen grupo de sectores mantienen ritmos de producción y exportaciones positivos. El consumo privado cae debido a la contracción experimentada por los salarios, la remuneración de capital y el número de empleados.

Las caídas experimentadas en las variables macroeconómicas son más intensas conforme mayores sean las barreras al comercio y a la IED que surjan con el Brexit. Si el Brexit supusiera las menores barreras que pueden darse (Brexit blando) el PIB caería un $0,32 \%$. En el extremo opuesto, la más intensa caída de $0,64 \%$ surgiría con las mayores barreras propias de un Brexit duro (sin acuerdo). Si se diera un Brexit con notable convergencia regulatoria en bienes y algo menor en servicios y sin aranceles con el resto de la UE (que es lo que modelizamos en el Brexit de Boris Johnson) las caídas podrían ser del 0,47\%. A título de referencia, si se dieran bajos aranceles en agricultura y alimentación y se ahorraran los trámites y costes de aduanas, en el hipotético caso de que Reino Unido permaneciese en la unión aduanera con la UE, como actualmente ocurre, la caída del PIB sería de un $0,52 \%$ anual.

Como ya se ha comentado, estas caídas del PIB son causadas por las solas fuerzas que el mecanismo del Brexit pondría en marcha, considerando todo lo demás constante. Las fuerzas del Brexit son de signo contractivo y reducirán aún más en las décimas que aparecen en el Cuadro 2 la contracción que la mayor parte de los organismos pronostica para el PIB español, tras el impacto del COVID, en 2021.

Es importante considerar que los distintos escenarios del Brexit los modelizamos con las barreras máximas que cabría esperar en cada uno de ellos. Reino Unido cumple actualmente con la legislación europea, por lo que una parte de las BNA, tanto al comercio como a la IED, se darán con el paso del tiempo. Sin embargo, los aranceles, si se dieran y las inspecciones en aduana junto con declaraciones en la misma, si salieran de la unión aduanera, lo que parece muy probable, surgirían desde el primer momento.

Ciertas BNA ligadas a nuevos procedimientos fiscales, contables, de protección de datos, de tratamiento de la propiedad intelectual, de obtención de certificados, licencias y autorizaciones, etc., surgirán desde el primer momento, junto con los costes ligados a la necesidad de elaborar planes de contingencia y de familiarizarse con el nuevo entorno por parte de empresas, administraciones y otros agentes económicos. Todo un nuevo entramado de relaciones posiblemente afectadas surgirá 
para las empresas (y muchas administraciones) relacionadas con clientes, proveedores, competidores, empleados, asesores, agencias reguladoras o autoridades competentes. El marco temporal en que todos estos factores se desarrollan no es sencillo de determinar y seguirá distinto curso en función del tipo de sector del que se trate. Por ello, nuestras estimaciones deben interpretarse como un máximo efecto cuando todas las fuerzas contractivas del Brexit estén en marcha. Mientras que el efecto del COVID cabe esperar que sea hondo, pero no tan duradero, en especial si se encuentra la vacuna, las barreras que surjan con el Brexit quedarán para siempre.

Los resultados del Cuadro 2 en concordancia con la mayor parte de los estudios realizados sobre el Brexit, señalan que los aranceles son el elemento que tiene un menor impacto cuantitativo. Su impacto, si finalmente se implementaran, sería automático y en el caso de los de nación más favorecida, tienen no desdeñables efectos en el agregado de las exportaciones y las importaciones.

El elemento de mayor importancia son las BNA aplicadas al comercio, si bien para el caso español es reseñable que el impacto de la IED le sigue a escasa distancia. Por supuesto, ambos componentes se encuentran modulados en función del tipo de acuerdo que se alcance y del consiguiente tamaño de las barreras que puedan surgir. Con mayores barreras, más intensa es la contracción en las distintas variables macroeconómicas. Aunque pocos estudios han cuantificado sus efectos, la sola fuerza de la IED en los sectores de servicios explica casi la mitad de la contracción del PIB en la mayoría de los escenarios del Brexit del Cuadro 2.

El Cuadro 2 recoge el impacto de un tratado ambicioso y modesto de la UE (incluyendo a España y a Reino Unido) con EEUU combinado con el Brexit de Johnson (con su impacto total, incluyendo tanto sus BNA como sus barreras a la IED). Es importante que en estos dos escenarios la situación de partida es la del impacto del propio Brexit de Johnson y que los resultados para los dos últimos bloques de resultados del Cuadro 2 indican hasta qué punto un tratado de toda la UE (incluyendo España y Reino Unido) con EEUU puede compensar el impacto negativo del Brexit de Johnson.

Este tratado con EEUU, si fuera ambicioso, compensaría los efectos negativos del Brexit, y, si fuera modesto, los suavizaría considerablemente. El Cuadro 2 muestra que el influjo aislado del componente de las BNA de un tratado ambicioso con EEUU compensaría en gran medida la caída del PIB provocada por el Brexit de Boris Johnson $(-0,47 \%)$, dejando el PIB en un $-0,10 \%$ (véase fila BNA del escenario Brexit de Johnson + Tratado ambicioso de Reino Unido y la UE con EEUU). Los aranceles y la IED de este tratado ambicioso con EEUU, de manera aislada, tienen menor impacto, y solo suavizarían un poco la caída del PIB del $-0,47 \%$ del Brexit de Johnson a $-0,43 \%$ (tras los aranceles) y a $-0,40 \%$ (tras la reducción de barreras a la IED). Cuando todos los componentes (BNA, aranceles e IED) entran en juego simultáneamente, del $-0,47 \%$ del impacto en el PIB de Johnson se pasa a un 0,03\% (fila «Total» del escenario Brexit de Johnson + Tratado ambicioso de Reino Unido y la UE con EEUU). Los patrones son similares, aunque de menor intensidad, para un tratado moderado con EEUU, tras el Brexit de Johnson. Las BNA serían las que más 
suavizarían la caída pasando de un $-0,47 \%$ a un $-0,35 \%$, que unidos a las menores aportaciones de aranceles e IED, acabarían dejando el PIB español, cuando todos los componentes interactúan simultáneamente, en una contracción del --0,27\%.

El Cuadro 2 refleja que el impacto del Brexit sería más bien limitado y, más aún, si lo combinamos con otros tratados comerciales en los que España esté involucrada. No obstante, hemos analizado también un escenario en un marco dinámico del modelo que consideramos poco probable pero que, si se diera, podría agravar considerablemente el impacto negativo. Si el Brexit desencadenara caídas fuertes en la inversión (doméstica o IED) en la economía, que acabaran provocando una reducción del stock de capital en nuestra economía, la contracción experimentada en nuestro país sería, aproximadamente el doble para los flujos comerciales, comparada con la del Cuadro 2 y alrededor del triple para el resto de variables macroeconómicas. Esto debe alertarnos respecto a la importancia de fomentar un clima favorable para la inversión cuando el Brexit sea implementado, algo especialmente delicado en el contexto del COVID, que de por sí está contrayendo la inversión agregada.

Por otra parte, el uso de un MEGA permite estimar el detalle de los impactos en la producción, empleo, salarios y exportaciones para cada uno de los 22 sectores del modelo junto con un total para el agregado de manufacturas, otro para el de servicios, así como el agregado de la producción del total de los sectores de la economía. A continuación, analizamos estos resultados, que subyacen a la evolución del PIB por el lado de la oferta.

\subsection{Resultados sectoriales}

El Cuadro 3 recoge el impacto en el total de las exportaciones españolas por sector y su evolución bilateral tras un Brexit duro, tras el Brexit propuesto por Boris Johnson, así como de este Brexit de Johnson combinado con un tratado del total de la UE28 (incluyendo por tanto a Reino Unido) con EEUU. Nos concentramos ahora en estos tres escenarios que ya hemos analizado a nivel macroeconómico para poder ofrecer un mayor elenco de resultados sectoriales. Los resultados que mostramos corresponden a la interacción simultánea de los componentes de comercio internacional y operaciones de multinacionales tanto en el Brexit como en el tratado con EEUU y se muestran como porcentajes de variación respecto al nivel inicial de las variables.

Empezaremos por un análisis del total de las exportaciones por sector. Varios sectores incrementan sus exportaciones y la caída máxima que experimentan las exportaciones sectoriales es de un 5.25\% en el Brexit duro del sector agrario. Se trata de un sector en el que Reino Unido tiene cierta importancia como destino (11,1\% de sus exportaciones se dirigen a las islas), y en el que las barreras esperables, tanto en forma de aranceles como de BNA, son de las más elevadas (sólo superadas por los aranceles en alimentación, ya que ambos registran los máximos en BNA al comercio). Con todo, detrás de la evolución del total del sector agrario hay cientos de 
empresas, que se verán más o menos afectadas por el Brexit en función de cuál sea su dependencia del mercado británico y su orientación exportadora. Tras el Brexit de Johnson las exportaciones de este sector caen un 3,43\%.

A las contracciones de agricultura siguen en importancia las de alimentación y automóvil tanto en el Brexit duro como en el de Johnson. El sector con mayor peso, tanto en las exportaciones como en las importaciones agregadas, a saber, química, está en el grupo de los que experimentan menores contracciones. Las exportaciones de otro sector de gran importancia, servicios a empresas prácticamente se mantienen, registrando una muy leve reducción en el Brexit de Johnson y un muy leve aumento en el duro. Otra maquinaria, que es también muy importante en el panorama de nuestras exportaciones agregadas también incrementa, aunque levemente sus exportaciones en los dos escenarios de Brexit. Otros sectores que aumentarían sus exportaciones son: construcción, transporte aéreo y comunicaciones.

Como ocurría a nivel macroeconómico, para entender el impacto de nuevos tratados o políticas comerciales tras el Brexit de Johnson no hemos de perder de vista que ahora la situación de partida, a diferencia del escenario de Brexit «aislado» de Boris Johnson, es la de las caídas en el comercio bilateral con Reino Unido generadas por el propio Brexit. Así, como aparece en el segundo bloque de resultados por columnas, tras el Brexit de Johnson, el comercio bilateral español con Reino Unido se contrae en un 18,20\%, a la par que se expande hacia la UE en un 0,60\% y hacia EEUU en un $0,68 \%$.

La combinación del Brexit de Johnson con un tratado ambicioso con EEUU firmado con toda la UE (incluyendo a España y Reino Unido) movería al terreno positivo al total de las exportaciones españolas (con un incremento del 0,18\%) si fuera ambicioso y las dejaría todavía en negativo $(-0,37 \%)$ pero compensando en gran medida, la mitad aproximadamente, el efecto contractivo del Brexit $(-0,83 \%)$ si fuera modesto. Tal y como aparece en la última fila del Cuadro 3 con el «Total de sectores» que, lógicamente, coinciden con las exportaciones agregadas del Cuadro 2 .

La mayoría de los sectores mejorarían sus exportaciones, comparado con el impacto asilado del Brexit de Johnson. Los sectores que más se benefician de este tratado serían alimentación, textil, química, metales y automóvil. Como todos ellos se encuentran entre los sectores más importantes para las exportaciones agregadas españolas, el impacto neto sería claramente positivo. Cierto que hay sectores que experimentan una mayor contracción en las exportaciones que las que el propio Brexit de Johnson genera, como ocurre con otro transporte y construcción y, en menor medida, con servicios a empresas, servicios personales y otros servicios. Construcción y otro transporte son sectores pequeños en el conjunto de las exportaciones. De los tres sectores de servicios, los servicios a empresas y otros servicios son de un considerable tamaño exportador. Sin embargo, el total de las exportaciones de servicios experimenta variaciones pequeñas comparadas con el Brexit de Johnson, ya que otros sectores de servicios mejoran sus exportaciones comparado con el Brexit de Johnson. 


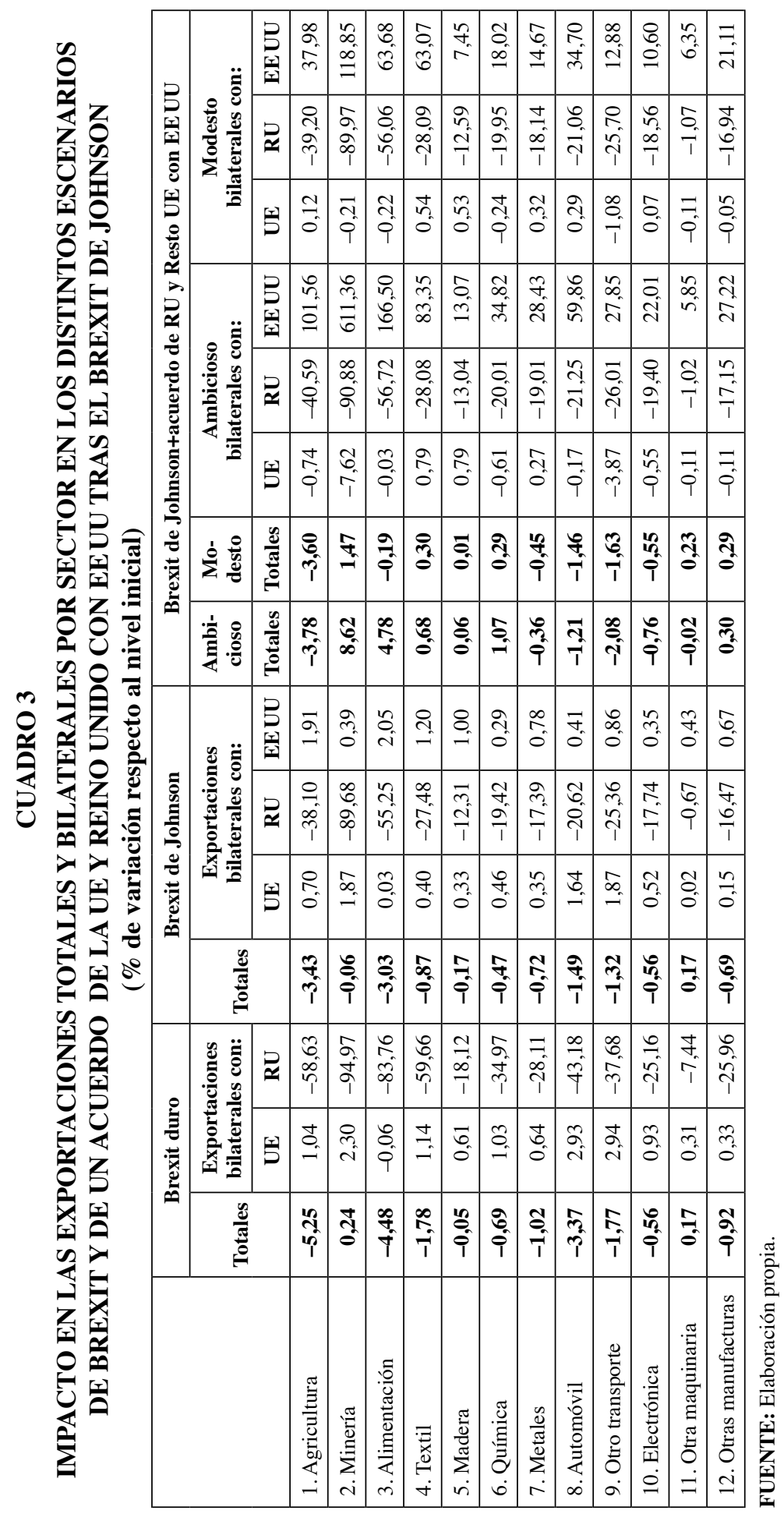




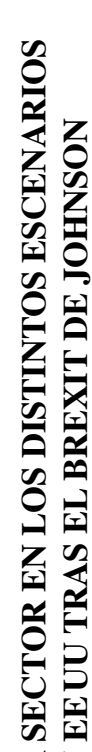

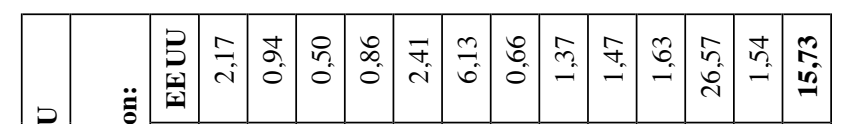

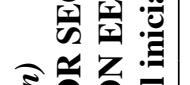

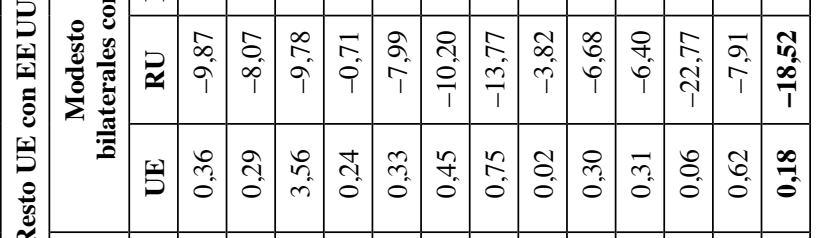

6

Z

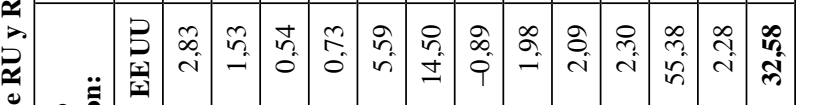

ชี้

:

空

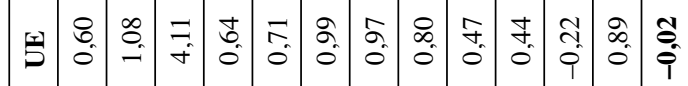

응

选兵

.

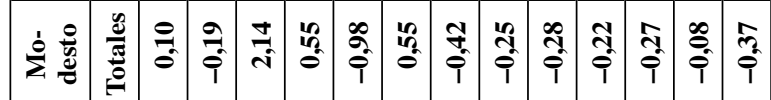

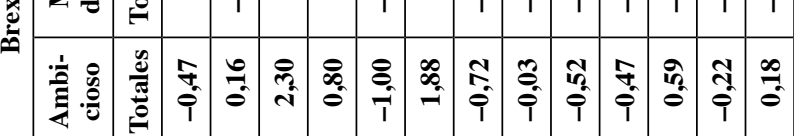

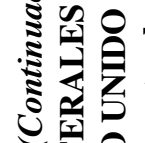

m民

舟里乐

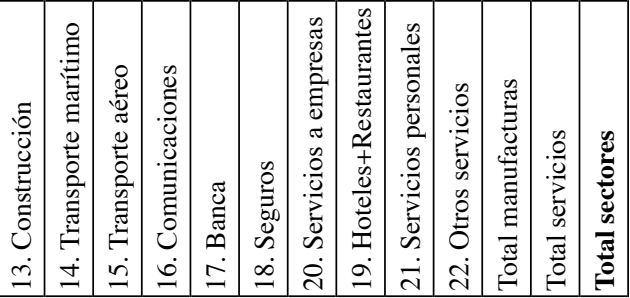


Pasando a analizar los datos de comercio bilateral recogidos en la Cuadro 3 observamos lo siguiente. El comercio bilateral español dirigido a la UE se expande tras el Brexit duro y el de Johnson. Sin embargo, como se deduce de las exportaciones totales que acabamos de analizar, esa expansión del comercio español hacia la UE no llega a compensar la contracción de nuestros flujos de comercio bilaterales con Reino Unido.

No obstante, la UE (y con ella España) puede encontrar una vía para compensar el Brexit negociando e implementando de manera efectiva nuevos acuerdos de comercio e IED, como el que presentamos con EEUU. Si pasamos a analizar las últimas seis columnas del Cuadro 3, volvemos al escenario de un tratado de toda la UE (incluyendo España y Reino Unido) con EE UU tras el Brexit. Con él, se daría una enorme expansión del comercio bilateral español con EEUU. De la situación tras el Brexit de Boris Johnson con un 0,61 \% de crecimiento, pasamos a un 32,58\% con las mayores reducciones de barreras posibles con EEUU (tratado ambicioso). Si fuera modesto, el incremento de nuestras exportaciones bilaterales con EEUU sería de un $15,73 \%$. Prácticamente todos los sectores aumentan sus exportaciones hacia el otro lado del Atlántico. Aparte del outlier del incremento del comercio bilateral con EEUU en minería, los mayores efectos expansivos hacia esa economía se registrarían en la alimentación, textil, agricultura y automóvil. Con todo, no hay que subestimar que las bajadas de barreras con EEUU aumentarían la competitividad de este país en el propio mercado interior de la UE, dificultando las exportaciones en el mercado único. Así, vemos que el comercio bilateral con respecto a la UE de España se mantendría (en realidad experimentan una muy ligera contracción del 0,02\%) tras un Brexit unido al tratado ambicioso con EEUU, frente al aumento del 0,60\% que experimentaba con el Brexit. Con reducciones de menor calado en las barreras transatlánticas (tratado modesto), el incremento de flujos bilaterales hacia la UE sería de $0,18 \%$, inferior al $0,60 \%$ del Brexit de Johnson. Los flujos hacia Reino Unido quedarían prácticamente iguales a los del Brexit de Johnson con caídas 18,66\% y $18,52 \%$ para un tratado ambicioso y modesto con EEUU, respectivamente, frente al $-18,20 \%$ del Brexit.

El Cuadro 4 recoge la evolución de la producción, el empleo y los salarios en todos los sectores. Como ocurría con los resultados del Cuadro 3 incluimos el impacto simultáneo de todos los componentes relacionados con el comercio y las operaciones de las multinacionales en estos cuadros sectoriales, expresados como porcentaje de variación respecto al nivel inicial.

Un buen grupo de sectores mantienen ritmos de producción positivos tras el Brexit de Johnson y el Brexit duro. Agricultura encabeza las contracciones, seguida del automóvil, hoteles y restaurantes y alimentación. Cabe señalar que ninguna de estas caídas, de los sectores más negativamente afectados supera el $2 \%$. La producción del sector agrario está notablemente orientada al mercado exterior $(32,8 \%$ se exporta) y, como ya señalamos, tiene una importante dependencia de Reino Unido. El automóvil encabeza la orientación exportadora de los sectores de la economía española, nada menos que un 78,5\% de su producción se destina a tal fin y, además, 
se encuentra también entre los que tienen mayor dependencia del mercado británico, pues un $13 \%$ de sus exportaciones se dirigen allí. Experimenta el surgimiento de barreras (aranceles y BNA) muy elevadas, aunque las máximas son las de agricultura y alimentación.

La evolución de las exportaciones supone una fuerza que, según su signo, tiende a impulsar o a frenar la producción sectorial. Con todo, no es la única fuerza en movimiento tras el Brexit, sino que la evolución del consumo privado y de la propia producción en el resto de sectores de la economía, junto con las barreras a la inversión productiva ligada a la IED, tienden a contraer la producción sectorial. Los sectores de servicios no se habían visto muy afectados negativamente en sus exportaciones, dado que su producción se orienta, en general, en mayor medida al consumo privado y a la provisión de servicios (inputs) intermedios para otros sectores. Sin embargo, la producción en los servicios está afectada más negativamente por el Brexit que las propias manufacturas, como podemos observar, comparando la evolución del «Total manufacturas» frente al «Total servicios» del Cuadro 4. De hecho, la evolución de los servicios, que contribuyen mucho más a la producción y el valor añadido español que las propias manufacturas, arrastra al «Total sectores» a unas caídas más en línea con la evolución de los servicios y más en línea, también, con la evolución del propio PIB de el Cuadro 2.

Cuando el Brexit de Johnson se combina con un tratado ambicioso de la UE (incluyendo España y Reino Unido) con EEUU, la producción mejora en la gran mayoría de los sectores. La excepción sería otro transporte y agricultura, que se verían afectados más negativamente que tras el propio Brexit. Otro transporte es un sector pequeño en el panorama nacional, pero la agricultura es de mayor entidad. Un proceso similar ocurre, en mucha menor medida, en electrónica y otra maquinaria. Para todos ellos habíamos visto mayores contracciones en las exportaciones tras el tratado con EEUU (siempre combinado con el Brexit de Jonhson) que con respecto al propio Brexit de Johnson «aislado». A pesar de que algunos sectores se ven afectados negativamente con el tratado con EEUU, el total de la producción agregada de la economía mejora, pasando de una caída con el Brexit de Johnson de 0,39\% a un $0,02 \%$. Por su parte, un tratado modesto, suaviza la caída provocada por el Brexit de Johnson, dejándola en una contracción de $0,24 \%$.

Los efectos sobre el empleo y los salarios de la última fila («Total sectores» de la economía) del Cuadro 4, coinciden, lógicamente, con los del empleo y los salarios a nivel macroeconómico recogidos en el Cuadro 2. Una clarificación importante es que, dado que la mitad de la mano de obra de cada sector es específica (es decir, sólo puede ser empleada en ese sector) y la otra mitad es móvil para desplazarse entre sectores, el salario del Cuadro 4 para cada sector es un agregado que comprende tanto la mano de obra específica como la móvil de ese sector. Por su parte, el empleo recoge la tasa de variación total en el empleo total del sector desde la situación previa al Brexit hasta la posterior al mismo.

El Cuadro 4 muestra que la evolución del empleo se mueve en paralelo con la de la producción en la mayoría de los sectores. Por lo general, aquellos sectores que 


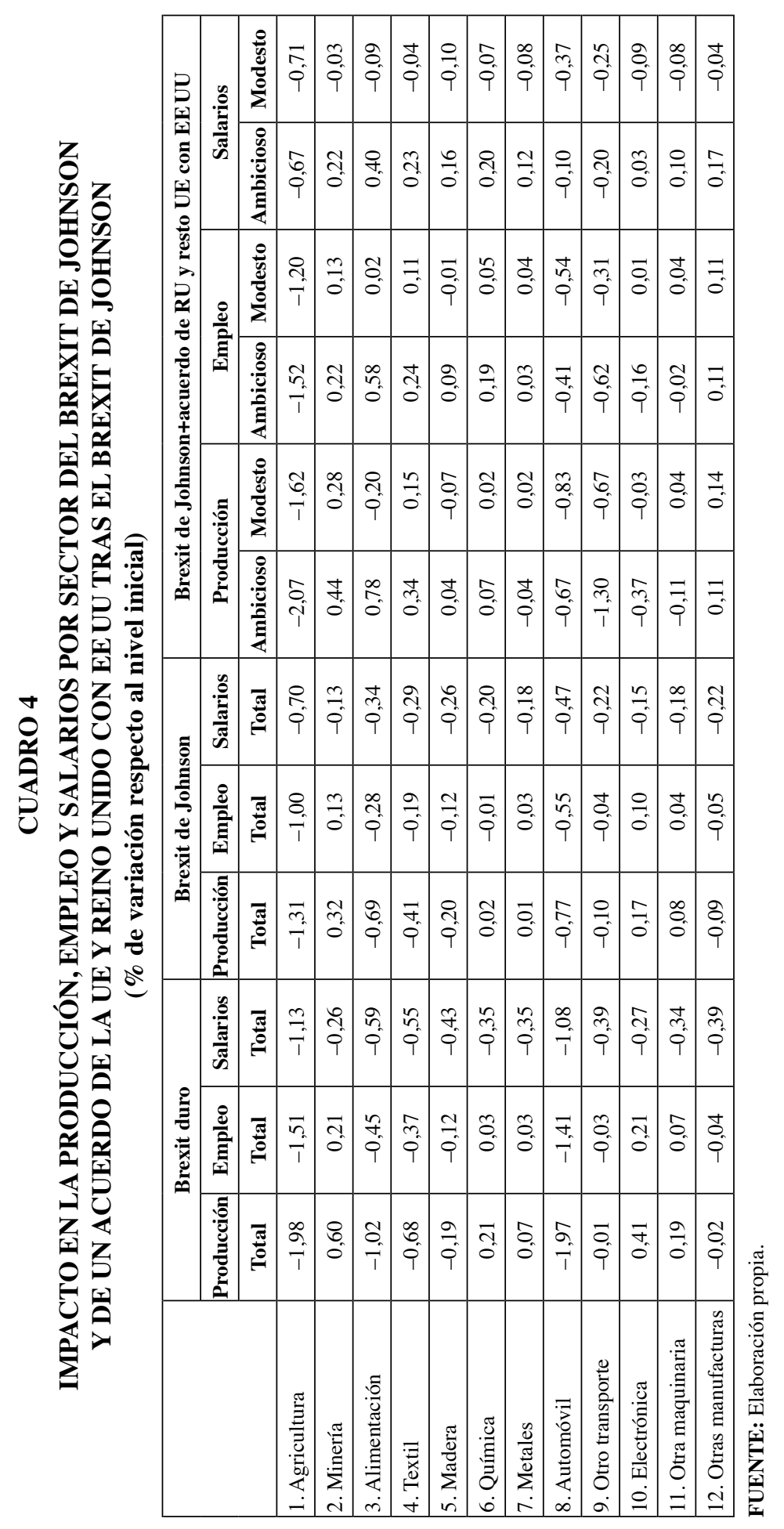




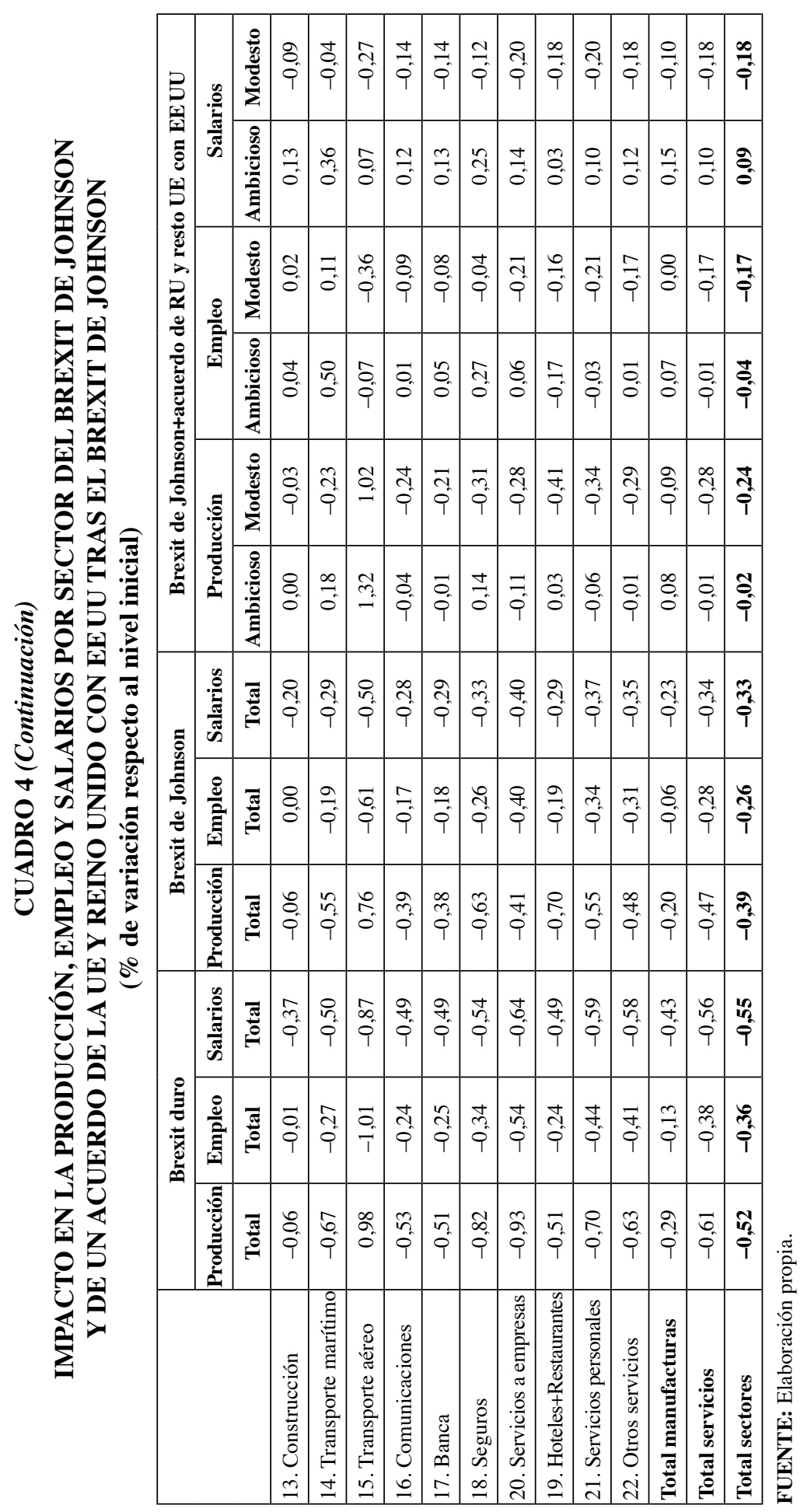


incrementan su producción elevan la demanda de mano de obra y los que reducen la producción, también disminuyen el número de empleados. Como ocurría también con la producción tras el Brexit duro y el de Johnson, ninguna de estas caídas del empleo supera el $2 \%$. La mayor de ellas, la de agricultura es menos intensa que un $2 \%$. Los sectores que mantenían su producción en positivo tienden a mantener también el empleo en positivo. La única excepción a este planteamiento es que el sector de Transporte aéreo que elevaba su producción reduce el empleo. En este caso, aunque la fusión entre British Airways e Iberia que dio lugar a la creación de IAG permita reorganizar la producción, en términos de empleo, las barreras del brexit suponen un coste que acaba pasando factura. Al ser un sector bastante intensivo en capital puede aumentar su producción sin tener que aumentar la mano de obra.

Los salarios caen en todos los sectores independientemente de que aumenten o disminuyan la producción y el empleo. Si bien, las caídas de salarios son ligeramente más pronunciadas en los sectores en los que la producción y el empleo están cayendo que en los que estas dos últimas variables se encuentran en positivo. Téngase en cuenta que tras el impacto contractivo del Brexit, que venimos analizando, aunque algunos sectores mantengan ritmos de producción positivos, los incrementos en la producción son moderados, de lo que se deriva que la generación de valor añadido generado (valor añadido = producción - consumo de intermedios) es menor que el habitual, lo que pone una presión a la baja a los salarios.

Como ocurría con la producción, las contracciones del empleo y los salarios que el Brexit de Johnson provoca se compensan en gran medida con un tratado con EEUU. Si fuera ambicioso, los salarios volverían al terreno positivo, elevándose respecto a los iniciales, y el empleo tendería a mantenerse (experimentando una muy leve contracción del $0.04 \%$ ). Con un tratado ambicioso, empleo y salarios en la mayor parte de los sectores conseguirían superar las contracciones del Brexit y acabarían expandiéndose. Un tratado modesto con EEUU, vuelve al terreno positivo el empleo en un buen número de sectores sin conseguir incrementos salariales en ningún sector. En el global el tratado modesto reduce el daño en la mayoría de los sectores, pero para el total de la economía tanto empleo como salarios permanecerían en terreno negativo, aunque suavizando las reducciones provocadas por el Brexit de Johnson.

\section{Conclusiones}

El uso de un modelo de equilibrio general aplicado (MEGA), nos ha permitido presentar un análisis muy detallado para distintas variables micro y macroeconómicas. Nuestro modelo es heredero del análisis de la introducción de Melitz (2003) en los modelos de equilibrio general (Balistreri et al., 2011) que hemos ampliado para modelizar las multinacionales siguiendo a Latorre y Yonezawa (2018). Se trata, por tanto, de un modelo avanzado que no solo incluye competencia monopolística à la Krugman (1980) para los sectores de servicios en los que hay multinacionales, 
sino además un clima de competencia monopolística à la Melitz (2003) en la mayor parte de los sectores manufactureros. Adicionalmente, para la modelización de la economía española hemos introducido la presencia de desempleo y fricciones en la movilidad de la mano de obra.

Nuestras estimaciones indican que el impacto negativo del Brexit en la economía española es limitado. Sin embargo, varían en función del tipo de relación futura entre Reino Unido y la UE, que entrará en vigor el 1 de enero de 2021, sin que aún se sepan buena parte de sus detalles. Ante las dificultades para alcanzar un acuerdo en un tiempo récord, junto con la tensión generada por el proyecto de la Ley del Mercado Interior del Reino Unido, no cabe perder de vista el más negativo de los escenarios, el del Brexit duro.

Conforme mayores son las barreras entre la UE y Reino Unido, más grande es el impacto negativo. Por tanto, el más dañino sería el Brexit duro, seguido del caso, muy poco probable, de que Reino Unido permaneciera en la Unión Aduanera con la UE, seguido, a su vez, del tratado de Johnson (con notable convergencia regulatoria en bienes y algo menor en servicios y sin aranceles con el resto de la UE), y el menor impacto tendría lugar tras un Brexit blando.

Es importante tratar de negociar una relación futura que haga surgir las menores barreras posibles. Si bien, es bueno tener presente, que el gran mercado para el comercio y la IED española es el europeo, incluso en la ausencia de Reino Unido, y que, si la negociación con los británicos hiciera peligrar el proyecto europeo, hay muchísimo más en juego con mantener el acceso sin barreras al mercado único que con las barreras que puedan surgir con Reino Unido.

Aunque pueda parecer lo contrario, el Brexit acaba siendo un movimiento proteccionista en lo que se refiere al comercio y a la IED con la UE. Puede que los obstáculos no lleguen a ser aranceles, pero eso tiene escasa entidad. Hoy en día los aranceles son de las barreras más pequeñas que se pueden poner al comercio.

El mercado único europeo ha logrado hacer de los regímenes de comercio e IED entre los distintos países europeos lo más similar que se puede encontrar en la faz de la tierra a operar dentro del mismo país. Ninguna zona del mundo, tiene regímenes tan similares entre países, ni el North American Free Trade Agreement (NAFTA, levemente modificado y rebautizado como United States-Mexico-Canada Agreement, USMCA), ni el Trans-Pacific Partnership (TPP, reconvertido en Comprehensive and Progressive Agreement for Trans-Pacific Partnership, CPTPP, tras el abandono de EEUU), ni un tratado como el Acuerdo Económico y Comercial Global suscrito entre la UE y Canadá, ni ningún otro tratado establece un régimen entre países con barreras tan bajas como el de la UE.

El Brexit supone poner trabas a las exportaciones españolas hacia las islas y a las operaciones de las multinacionales españolas en Reino Unido y de las británicas en España. Este trabajo ofrece estimaciones para las contracciones que experimentarían la producción, el empleo y los salarios en España.

Las barreras no arancelarias (BNA) al comercio son el principal foco de las tendencias contractivas después del Brexit. Este es un resultado en línea con la mayor 
parte de los estudios realizados sobre el Brexit. Por eso afirmábamos que si el tratado que se acuerde entre Reino Unido y la UE se centra solo en la eliminación de los aranceles (y cuotas que están incluidas también en el cálculo de los aranceles de nuestro modelo), cabe esperar que con el tiempo surgirán importantes BNA, relacionadas con las divergencias regulatorias entre la UE y Reino Unido.

Con todo, el impacto de las barreras a la IED también es muy importante en la economía española. Las multinacionales extranjeras que operan en sectores de servicios avanzados son proveedoras de servicios intermedios necesarios para la producción en otros sectores y de distintas variedades de servicios finales para los consumidores que han pasado casi desapercibidas en la literatura del Brexit. En el caso español, esta sola fuerza relacionada con la IED, sin contar con las contracciones ligadas al comercio internacional, explica casi la mitad del impacto negativo del Brexit a nivel macroeconómico. El efecto de las multinacionales en España tras el Brexit, es mayor que en el propio Reino Unido (Latorre et al., 2020a; 2020b). Las economías son crecientemente un sistema productivo de bienes y servicios interrelacionados de maneras cada vez más complejas (Baldwin, 2016).

Un tratado ambicioso de la UE (incluyendo a España y a Reino Unido) con EEUU compensaría los efectos negativos del Brexit en la economía española, y si fuera modesto, los suavizaría considerablemente. Hemos ilustrado el impacto conjunto del Brexit de Boris Johnson con este tratado con EE UU tanto a nivel sectorial como macroeconómico. La mayor parte de los sectores de la economía española se beneficiarían. La UE con su enorme experiencia en la negociación de tratados debe seguir explorando nuevas posibilidades. Pues, tal y como podemos ver con los resultados con EEUU, estos tratados brindan numerosas posibilidades del crecimiento, creación de empleo y mejoras salariales.

\section{Referencias bibliográficas}

Aguiar, A., Chepeliev, M., Corong, E. L., McDougall, R., \& van der Mensbrugghe, D. (2019). The GTAP Data Base: Version 10. Journal of Global Economic Analysis, 4(1), 1-27.

Balistreri, E. J., Hillberry, R. H., \& Rutherford, T. F. (2011). Structural estimation and solution of international trade models with heterogeneous firms. Journal of International Economics, 83(2), 95-108. https://doi.org/10.1016/j.jinteco.2011.01.001

Bisciari, P. (2019). A survey of the long-term impact of Brexit on the UK and the EU27 economies (No. 366). National Bank of Belgium Working Paper.

Böhringer, C., Löschel, A., Moslener, U., \& Rutherford, T. F. (2009). EU climate policy up to 2020: An economic impact assessment. Energy Economics, 31(Suppl. 2), S295-S305. https://doi.org/10.1016/j.eneco.2009.09.009

Busch, B., \& Matthes, J. (2016). Brexit - the economic impact: A meta-analysis. IW Report

Baldwin, R. E. (2016). The Great Convergence: Information Technology and the New Globalization. Harvard University Press. 
Burfisher, M. E. (2011). Introduction to computable general equilibrium models. Introduction to computable general equilibrium models. Cambridge University Press. https://doi.org/10.1017/CBO9780511975004

Dhingra, S., Huang, H., Ottaviano, G., Pessoa, J. P., Sampson, T., \& Van Reenen, J. (2017). The costs and benefits of leaving the EU: Trade effects. Economic Policy, 32(92), 651705. https://doi.org/doi:10.1093/epolic/eix015

Dixon, P. B., \& Jorgenson, D. W. (2013). Introduction. En P.B. Dixon and D.W. Jorgenson (Eds.), Handbook of CGE Modeling, (pp. 1-22). Elsevier.

Eurostat (2019). Foreign control of enterprises by economic activity and a selection of controlling countries (from 2008 onwards).

http://appsso.eurostat.ec.europa.eu/nui/show.do?dataset=fats_g1a_08\&lang=en

Felbermayr, G., Gröschl, J. K., \& Steininger, M. (2018). Quantifying Brexit: From Ex Post to Ex Ante Using Structural Gravity (No. 7357). Center for Economic Studies and Ifo Institute, CESifo Working Paper. https://ideas.repec.org/p/ces/ceswps/_7357.html

Fernández-Pacheco Theurer, C., López-Ruiz, J. L., \& Latorre, M. C. (2018). Multinationals' effects: A nearly unexplored aspect of Brexit. Journal of International Trade Law and Policy, 17(1-2), 2-18. https://doi.org/10.1108/JITLP-12-2017-0053

Francois, J., Manchin, M., Norberg, H., Pindyuk, O. \& Tomberger, P. (2013). Reducing Transatlantic Barriers to Trade and Investment, An Economic Assessment. Study for the European Commission, CEPR Report.

Gov UK (2019). Revised Political Declaration, 18 de octubre. https://assets.publishing. service.gov.uk/government/uploads/system/uploads/attachment_data/file/840231/ Revised_Political_Declaration.pdf

Hurtado, S. (2019). Actualización de los efectos estimados para la economía española de la salida del Reino Unido de la UE. Nota interna, octubre de 2019, Banco de España.

Jafari, Y., \& Tarr, D. G. (2017). Estimates of ad valorem equivalents of barriers against foreign suppliers of services in eleven services sectors and 103 countries. World Economy, 40(3), 544-573. https://doi.org/10.1111/twec.12329

Krugman, P. (1980). Scale economies, product differentiation, and the pattern of trade. American Economic Review, 70(5), 950-959.

Latorre, M. C., \& Yonezawa, H. (2018). Stopped TTIP? its potential impact on the world and the role of neglected FDI. Economic Modelling, 71, 99-120. https://doi.org/10.1016/j.econmod.2017.12.006

Latorre, M. C., Yonezawa, H., \& Zhou, J. (2015). El impacto del TTIP en la economía española. Instituto de Estudios Económicos.

Latorre, M. C., Olekseyuk, Z., Yonezawa, H., \& Robinson, S. (2019). Brexit: Everyone loses, but Britain loses the most (No. WP19-5) Peterson Institute for International Economics (PIIE).

https://piie.com/publications/working-papers/brexit-everyone-loses-britain-loses-most

Latorre, M.C., Olekseyuk, Z., Yonezawa, H., \& Robinson, S. (2020a). Making sense of Brexit losses: An in-depth review of macroeconomic studies, Economic Modelling, 89 (C), 72-87. https://doi.org/10.1016/j.econmod.2019.10.009

Latorre, M. C., Yonezawa, H., \& Olekseyuk, Z. (2020b). Trade and Foreign Direct Investment-related impacts of Brexit. World Economy, 43(1), 2-32.

https://doi.org/10.1111/twec.12859 
Melitz, M. J. (2003). The impact of trade on intra-industry reallocations and aggregate industry productivity. Econometrica, 71(6), 1695-1725. https://doi.org/10.1111/1468-0262.00467

Shoven, J. B., \& Whalley, J. (1992). Applying General Equilibrium. Cambridge University Press.

Vega, J. L. (Coord.) (2019). Brexit: balance de situación y perspectivas. Documentos ocasionales n. ${ }^{\circ}$ 1905, marzo de 2019, Banco de España. 